\title{
Congenital Infantile Intracranial Teratoma with Orbital Extension
}

\section{Muhammad Rafay, Muhammad Yousuf Shaikh ${ }^{*}$ and Salman Sharif}

Department of Neurosurgery, Liaquat National Hospital, Karachi, Pakistan

“Corresponding author: Muhammad Yousuf Shaikh, Department of Neurosurgery, Liaquat National Hospital, Karachi, Pakistan, Tel: 923223302593; E-mail: unknownyou@hotmail.com

Received date: October 22, 2018; Accepted date: November 1, 2018; Published date: November 8, 2018

Copyright: (c) 2018 Rafay M, et al. This is an open-access article distributed under the terms of the Creative Commons Attribution License, which permits unrestricted use, distribution and reproduction in any medium, provided the original author and source are credited.

\begin{abstract}
Background: Mature intracranial teratoma in the pediatric population is a rare entity. They are sometimes associated with genetic syndromes but complete surgical excision is associated with good outcome and prognosis.

Case Presentation: A 3 months old baby girl presented in the clinic with left eye proptosis, MRI brain showed temporal and retro-orbital multiple cystic lesions, which were excised and biopsy showed mature teratoma. The patient was discharged and followed in the clinic.

Conclusion: This case underlies that mature brain teratoma may present at an early age even at birth. They are rare intracranial tumors and may be associated with other genetic syndromes. A high index of suspicion should be present when coming across such complex lesions in neonates. Although the overall outcome of these tumors is generally poor, among the children that survive, complete microsurgical excision of the tumor is associated with good outcome and prognosis. In cases of mature teratomas, we advocate that complete removal should be carried out. In cases of the residual disease, reoperation is indicated to achieve good results.
\end{abstract}

Keywords: Biopsy; Tumor; Teratoma, Genetic syndrome

\section{Introduction}

Teratomas are tumors originating from misplaced embryonic germ cells. The heterogeneity of tissues they contain indicates their origin from more than one germ cell layer. Radiological diagnosis is often difficult as they tend to present as multi-loculated cystic masses with complex radiological findings. Clinical manifestation may vary from simple enlargement of the skull to life-threatening issues attributed to hydrocephalus, respiratory distress or feeding difficulties. In infancy, these tumors can behave as biologically benign tumors leading to good outcome after total resection [1].

\section{Case Presentation}

A 3 months old girl was brought in Neuro-Surgical OPD with Left eye proptosis and malformed left ear since birth. MRI brain with contrast was done which showed multiple cystic lesions involving left temporal region, extending up to left retro-orbital area causing forward displacement of the left eyeball (Figure 1). Optic nerve and recti muscle seemed inseparable and the lesion was causing erosion of the greater and lesser wings of the sphenoid, raising the suspicion of lymphangiomas. She underwent craniotomy and biopsy of the retroorbital lesion which showed benign mucinous glands with respiratory epithelium. Post-procedure she developed $6^{\text {th }}$ nerve palsy. The parents were counseled about total resection of the lesion after which they lost to follow up.

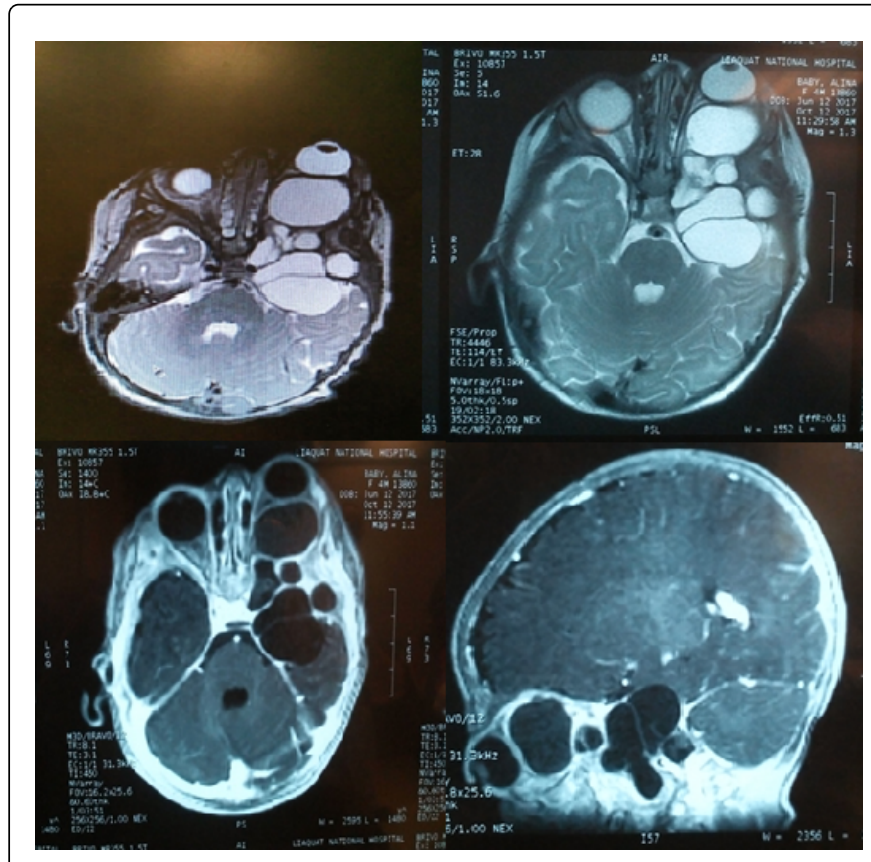

Figure 1: Pre-operative MRI showing multiple cystic lesions in left temporal and retro-orbital area. 
At the age of 9 months, she was brought to our Clinic and she subsequently underwent craniotomy and excision of the tumor. Intraoperatively, tumor cavity was filled with thick mucinous type material (Figure 2). The tumor was completely removed and a large surgical cavity left behind which was filled with glue and artificial Dura. Postoperatively her proptosis improved (Figure 3). Postoperative MRI scan was done on the $7^{\text {th }}$ day which showed a suspected residual lesion in the temporal region (Figure 4). Biopsy turned out to be mature teratoma containing, mucinous glands, skeletal tissue, and adipose tissue with no evidence of any immature components. The patient was discharged without any complications and followed in clinics regularly. She is now planned for removal of residual disease.

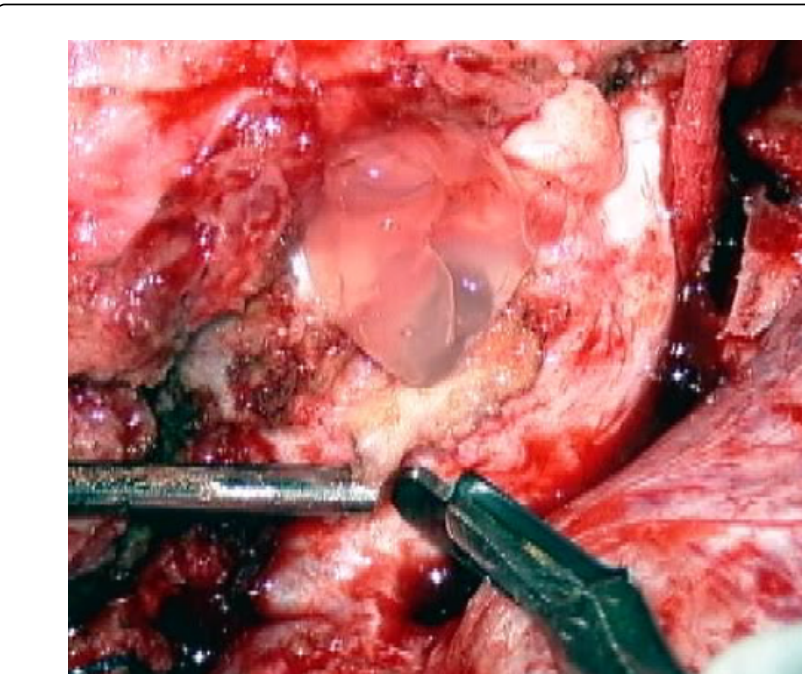

Figure 2: Intra-operative picture showing jelly-like material being aspirated from the cyst.

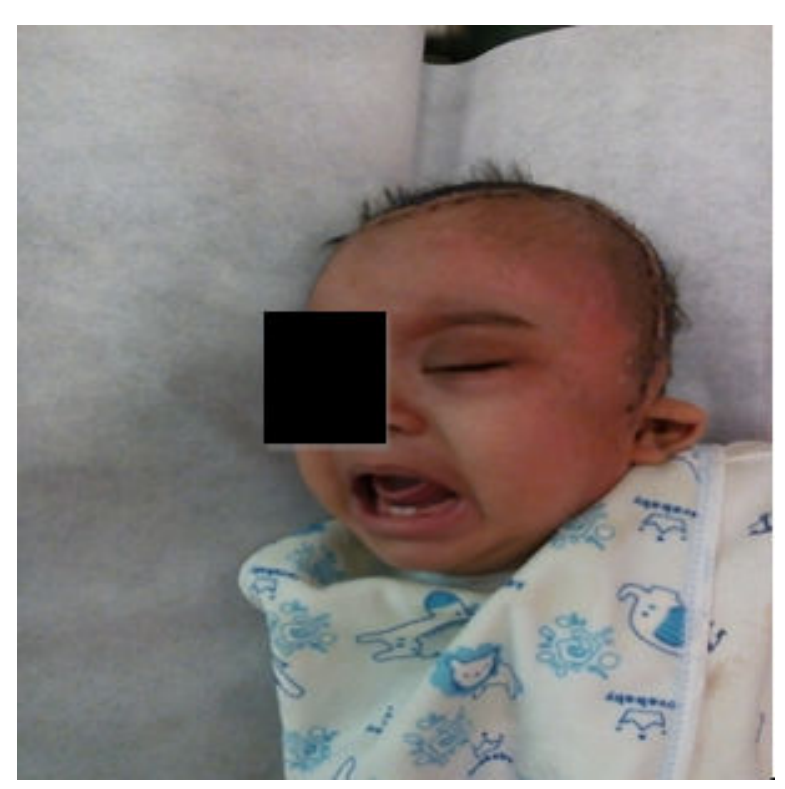

Figure 3: Post-operative picture.

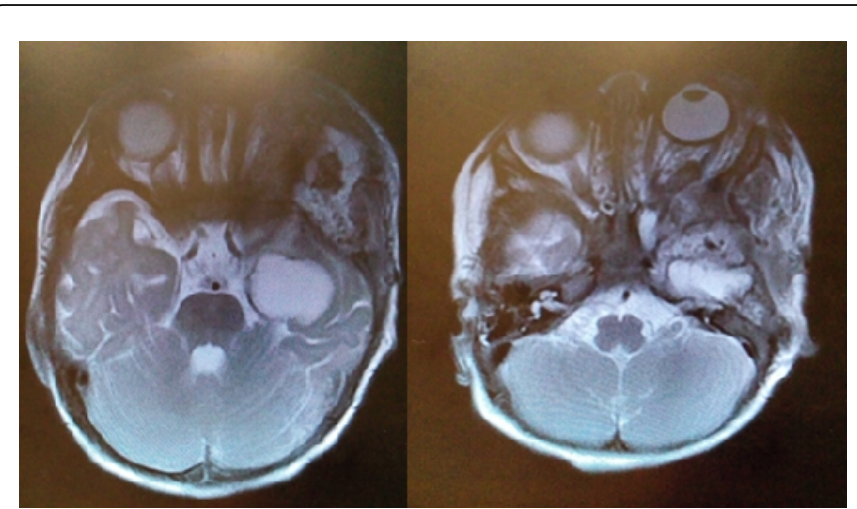

Figure 4: Post-operative MRI T2 screening showing a suspicious area of residual disease in the left temporal lobe.

\section{Discussion}

Congenital intracranial tumors are those which we are able to diagnose during pregnancy or within 3 months after birth [2]. The reported incidence of Childhood brain tumors is $0.5 \%-1.5 \%$ of all childhood tumors. [3] These tumors can either be solid or cystic. Among the solid tumors, Teratomas are relatively common solid neoplasms in children. They can arise from the pineal gland, quadrigeminal plate or walls of the third ventricle, suprasellar region or cerebellar vermis. Occasionally it is not possible to identify the exact site of origin of such tumors but generally speaking, prenatally diagnosed tumors are supratentorial while older children tend to present with infratentorial teratomas [4]. Teratomas may occasionally contain cystic spaces which may make diagnosis difficult. In such circumstances, arachnoid cysts should be considered as another possibility. Occasionally the MRI may show areas of calcification as well. But in all circumstances, it is important to excise the lesion and get histological diagnoses.

Clinical presentation of these tumors is a bit variable. But the degree of immaturity plays an important role in the presentation of such lesions as well as the outcome. Majority of neonates with congenital teratomas are either born stillbirth or die soon after birth. Histological studies of these tumors on autopsy usually indicate immature elements which correlate with significantly poor outcome. As a result, reported mortality for such lesions has been estimated to be as high as $90 \%$ [5]. On the contrary, mature teratomas have low recurrence rates on account of containing mature elements. These may have significantly better outcome following complete surgical removal.

Radiologically, teratomas usually present as large heterogeneous masses with a variable amount of solid and cystic masses. A CT Scan will show missed density lesion with hypodense fatty components and hyperdense calcified portion indicating teeth or bone [6]. The presence of fat, calcification and solid components is highly suggestive of a teratoma. MRI Brain with contrast will also show similar characteristics depending on the content of tumor with mixed hyper to hypointense signals.

Only a few cases of congenital intracranial teratomas have been reported in history so far making them a relatively rare entity. One such case of congenital intra-cranial teratoma is reported by Enrique $\mathrm{C}$ et al. where they successfully removed the entire tumor from neonate [7]. They also advocated that the outcome and long-term survival 
depends on the maturity of the lesion. Recurrent lesions need reoperation in benign cases whereas immature malignant tumors respond to chemotherapy and radiotherapy. In one of the recent case report by Eric Moreddu et al. [8], they successfully removed a large intracranial teratoma with orbital and pharyngeal extension using a combined open and endonasal technique. Masao Matsutani et al. analyzed 153 cases of histologically verified intra-cranial germ cell tumors with up to 10 years follow up. They reported 10 year survival rates of up to $92.9 \%$ with mature teratomas [9].

Overall, intracranial teratomas represent a rare entity. And this accounts for their variable radiological as well as clinical presentation. Since the majority of such lesions are immature, they grow rapidly as such that they may occasionally completely replace normal brain leading to stillbirths or death soon after birth. But in circumstances where the neonate survives, early surgery is indicated to remove the maximum amount of tumor and achieve a histological diagnosis. Because of the complex radiological findings, the index of suspicion should always be high when coming across such complex lesions in neonates, because complete removal of mature teratomas is curative.

Dealing with recurrence or residual disease is also a challenging task. Although in cases of immature teratomas, redo surgery may not be very helpful because of the high mortality associated with these lesions, mature teratomas should always be re-explored and planned total resection done. As in our case, we had a residual disease which we plan to address in coming few weeks.

\section{Conclusion}

This case underlines that mature brain teratoma may present at an early age even at birth. They are rare intracranial tumors and may be associated with other genetic syndromes. A high index of suspicion should be present when coming across such complex lesions in neonates. Although the overall outcome of these tumors is generally poor, among the children that survive, complete microsurgical excision of the tumor is associated with good outcome and prognosis. In cases of mature teratomas, we advocate that complete removal should be carried out. In cases of the residual disease, reoperation is indicated to achieve good results.

\section{Acknowledgment}

Written consent was obtained from the patient for publication of the patient's details.

\section{References}

1. Smirniotopoulos JG, Chiechi MV (1995) Teratomas, dermoids, and epidermoids of the head and neck. Radiographics 15:1437-1455.

2. Alamo L, Beck-Popovik M, Gudinchet F, Meuli R (2011) Congenital tumors: Imaging when life just begins. Insights imaging 2: 297-308.

3. Catherine G (2004) Intracranial space occupying lesions. MRI of the fetal brain book. Berlin Heidelberg, Springer-Verlag, USA, pp: 177-200.

4. Buetow PC, Smirniotopoulos JG, Done S (1990) Congenital brain tumors: A review of 45 cases. Am J Roentgenol 155: 587-593.

5. Isaacs H Jr. (2002) I. Perinatal brain tumors: A review of 250 cases. Pediatr Neurol 27: 249-61.

6. Tortori-Donati P, Rossi A, Biancheri R, Garre ML, Cama A (2005) Brain tumors, (Edn 1), J Pediatr Neuroradiol, Springer, Berlin, pp: 329-436.

7. Ventureyra EC, Herder S (1991) Neonatal intracranial teratoma. Case report. J Neurosurg 59: 879-883.

8. Moreddua E, Pereirab J, Vazb R, Lenac G, Trigliaa JM (2015) Combined endonasal and neurosurgical resection of a congenital teratoma with pharyngeal, intracranial and orbital extension: Case report, surgical technique, and review of the literature. Int J Pediatr Otorhinolaryngol 79: 1991-1994.

9. Matsutani M, Sano K, Takakura K, Fujimaki T, Nakamura O, et al. (1997) Primary intracranial germ cell tumors: A clinical analysis of 153 histologically verified cases. J Neurosurg 86:446-455. 